\title{
ESTRUCTURA DE AUTORIDAD Y RELACIONES DE GÉNERO entre los lanoh de la península de Malasia
}

\section{Csilla Dallos}

Assistant professor, Departament of Anthropology,

\author{
St. Thomas University
}

dallos@stu.ca

\section{Resumen}

[ STE ARTÍCULO ANALIZA LAS RAZONES POR LAS CUALES LA SEPARACIÓN ENTRE EL DOMINIO [ público y el privado en una aldea lanoh, de la península de Malasia, cuya población está formada por recolectores que recientemente adoptaron la vida sedentaria, no resultan en las desigualdades de género pronosticadas por el modelo de Rosaldo. La conservación de la autonomía individual entre los lanoh evita que los líderes que compiten por el poder obtengan apoyo de sus parientes masculinos. En consecuencia, su auto-glorificación queda confinada al ámbito de sus hogares. Las desigualdades de género en la aldea sólo se dan en los hogares nucleares de los líderes, como consecuencia del aumento de la producción familiar. Nuestros resultados nos llevan a sostener que las causas de las desigualdades en sociedades de pequeña escala deben ser investigadas tomando en consideración distintos tipos de relaciones, no sólo las que privan entre los hombres y las mujeres.

PALABRAS CLAVE: género, inequidad, cazadores-recolectores, Malasia.

\section{Abstract}

T HIS ARTICLE CRITICALLY EXAMINES WHY THE SEPARATION OF PUBLIC AND PRIVATE DOMAINS IN a village of newly sedentary Lanoh forest collectors and traders in Peninsular Malaysia does not result in the gender inequality predicted by Renato Rosaldo's influential model. Continuing individual autonomy among the Lanoh prevents competing leaders from attaining support from their male kin. As a result, their self-aggrandisement is confined to their nuclear households where they are supported by their female kin. Gender inequality in the village only occurs in these leaders' nuclear households, as a consequence of increased household production. These findings imply that inquiries into the causes of gender inequality in small-scale societies need to examine relationships other than those between men and women.

KEY wORDS: Gender, inequality, hunter-gatherers, Malaysia.

$$
\frac{\text { Revista Colombianade Antropología }}{\text { Volumen 42, enero-diciembre 2006, pp. } 99-128}
$$




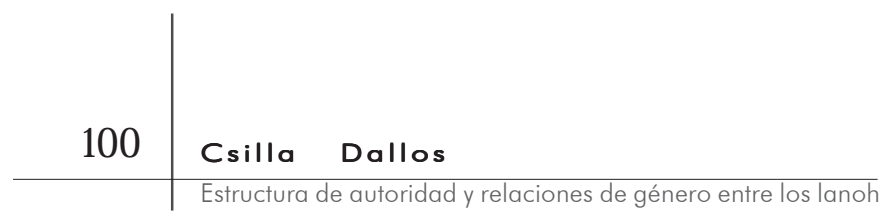

\section{INTRODUCCIÓN}

C NCONTRAR LAS RAÍCES DE LA DESIGUALDAD DE GÉNERO HA PERDIDO PO- pularidad como objetivo de investigación en antropología. Des- de las décadas de 1980 y 1990, una vez logrado el consenso acerca del rol de los significados culturales en la determinación de las relaciones de género en diferentes ámbitos sociales (Collier y Yanagisako, 1987; Di Leonardo, 1991), la investigación feminista abandonó la búsqueda de los orígenes de la desigualdad de género, a medida que los trabajos deconstruccionistas se apartaban de las grandes théories y de los estudios comparativos, y se orientó hacia el estudio de las diferencias culturales y de la subjetividad (Lewellen, 2003).

Este giro fue tal vez prematuro, pues la teoría que explica las relaciones de género a partir de significados culturales es insatisfactoria y comporta múltiples problemas. En consecuencia, basarse en dicha teoría lleva a perpetuar concepciones erróneas acerca del género, como por ejemplo, la que asume que las ideologías de género se derivan de diferencias biológicas entre los hombres y las mujeres. De hecho, puede argüirse que la base para diferenciar entre factores biológicos y culturales, sexo frente a género, sirve para defender la hipótesis según la cual la dominación masculina se genera por significados culturales, de manera similar a como las diferencias sexuales, o sea biológicas, se interpretan en diferentes contextos sociales. Esta es una explicación conveniente pero que carece de fundamento, dado que los significados no siempre enfatizan en diferencias biológicas reales entre hombres y mujeres (Dallos, 2003). Al contrario, al examinarlas detenidamente, estas diferencias se revelan como convenientes, atributos ficticios, masculinos y femeninos, que, en muchos casos, tienen poco que ver con diferencias sexuales.

A veces, el vínculo entre los significados culturales y las diferencias biológicas es resultado de nuestras propias interpretaciones antropológicas. Por ejemplo, en un conocido modelo de diferencias de género, la dicotomía que considera a la mujer como "natural” y al hombre como "cultural" surgió como resultado de la interpretación de significados locales hecha por Orner, considerándolos en términos de diferencias sexuales en las funciones reproductivas (1974). Además, cabe afirmar que los significados no producen discriminación por sí mismos. A pesar 


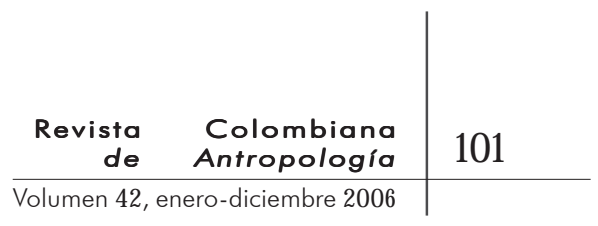

de que los cazadores-recolectores consideran con frecuencia que durante la menstruación las mujeres traen mala suerte en la cacería, esto no implica ni maltrato ni explotación en esas sociedades, igualitarias en los demás aspectos, porque entre ellos no existen las relaciones sociales que convertirían esta creencia en desigualdad de género. Por todo lo anterior, es tarea fundamental comprender las relaciones sociales que subyacen a la desigualdad de género.

Es aquí donde encontramos un obstáculo teórico adicional: una definición que no nos permitiría estudiar diferentes grados de desigualdad social. Según Yaganisako y Collier:

\begin{abstract}
Partimos de la premisa de que los sistemas sociales son, por definición, sistemas de desigualdad. Esta premisa (...) es de uso común. De acuerdo con la mayor parte de definiciones, una sociedad es un sistema de relaciones sociales y valores. Todo valor supone evaluación. En consecuencia, una sociedad es un sistema de relaciones sociales en el que ni las cosas ni las acciones son iguales (1987: 39).
\end{abstract}

Es posible que esta inferencia sea de uso común, pero infortunadamente es poco útil para el estudio de la desigualdad social. Si la desigualdad fuera sinónimo de diferencias de valores y dichas diferencias hechos universales, entonces no tendría sentido distinguir entre grados de desigualdad social. Obviamente, este no es el caso. Así mismo, si consideráramos las desigualdades como diferencias de valores, entonces la desigualdad social se volvería un concepto antropocéntrico. Entre animales, por ejemplo, no existen diferencias de valores. ¿Significa esto, acaso, que en los grupos sociales de animales priva la igualdad?

No pretendemos con esto negar la utilidad de los términos sexo y género en antropología. Es posible, por ejemplo, continuar usándolos al tratar de distinguir entre las diferencias biológicas que distinguen a los hombres y a las mujeres y sus percepciones culturales. Sin embargo, en vez de seguir dilucidando significados, deberíamos enfocarnos en personas reales, en mujeres involucradas en relaciones sociales; en mujeres discriminadas, maltratadas, atacadas, hasta quemadas vivas, en el contexto de la desigualdad de género. No tiene mucha importancia determinar si estas personas son mujeres en un sentido sexual o de género. Lo que se vuelve crucial es comprender cómo los grupos humanos se transforman, llegando a permitir que se 


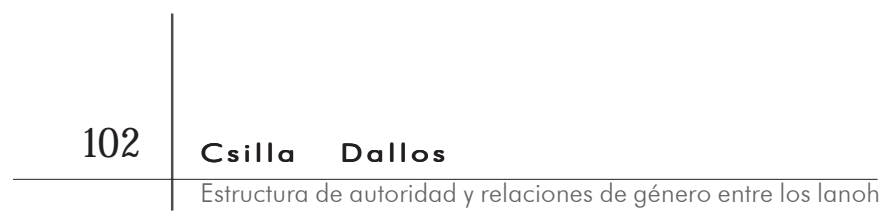

cometan tales abusos contra una fracción importante de su población.

Por tanto, podemos definir el estudio de la desigualdad de género como el de cómo percepciones aparentemente inofensivas se transforman en acciones que pueden llegar a tener serias consecuencias. Para que eso suceda es preciso que las sociedades cambien. De acuerdo con Lewellen, "el análisis estructural, por sí solo, no es suficiente para explicar la subordinación femenina. La estratificación -ya sea de estatus, rango, clase o género-, se produce dentro de la historia" (2003 150-157). El estudio de la desigualdad de género no puede disociarse del estudio del cambio social. Las teorías antropológicas y arqueológicas sostienen que la sedentarización es uno de los cambios más importantes que un grupo social puede experimentar. En consecuencia, dilucidar la relación entre sedentarismo y desigualdad de género se ha convertido en un aspecto importante del estudio de los efectos del cambio social sobre las relaciones de género (Draper, 1975a, b, 1992; Kent, 1995a, b).

Todos los estudios mencionados se basan en la premisa de que los grupos de cazadores-recolectores que recientemente se han vuelto sedentarios pueden enseñarnos acerca de las relaciones sociales que resultan de la sedentarización, y que este conocimiento puede aplicarse en diferentes circunstancias. Desde la década de 1990, autores revisionistas han cuestionado que los modelos basados en cazadores-recolectores puedan explicar procesos históricos y prehistóricos (Grinker, 1992; Kelly, 1995 , Shott, 1992). A pesar de la validez de dichas críticas, espero demostrar que los cambios experimentados por poblaciones modernas de cazadores-recolectores pueden ser útiles para comprender procesos de cambio, durante periodos históricos y, quizá, hasta prehistóricos.

Aun cuando los estudios de Draper (1975, b, 1992) y Kent (1995a, b) aumentaron considerablemente nuestra comprensión de las relaciones de género entre cazadores-recolectores recientemente sedentarizados, sus conclusiones son parciales. En sus artículos, Draper identifica los patrones de subsistencia y movilidad, la división sexual del trabajo, el tamaño del grupo, los patrones de asentamiento (1975), y, finalmente, las relaciones con grupos vecinos, como factores que ejercen influencia sobre las relaciones de género de los grupos que se vuelven sedentarios. Kent (1995a) sugiere que es poco probable que la sedentarización, por 


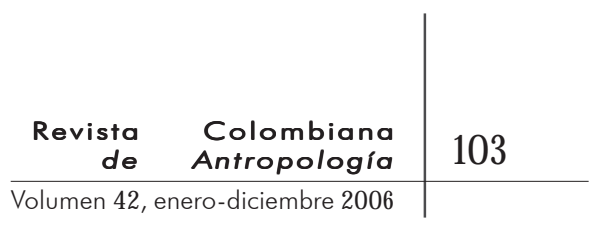

sí sola, produzca desigualdad de género, enfatizando en la aculturación y los efectos de los valores de los agricultores vecinos sobre las relaciones de género entre los grupos de cazadoresrecolectores recientemente sedentarizados. Al referirse a las situaciones iniciales, especula sobre las fuentes de inequidad en ausencia de influencias externas (1995a).

En este artículo trato estos aspectos, con interrogantes similares, pero con énfasis en los efectos que los patrones demográficos cambiantes y de asentamiento pueden tener sobre las relaciones de género. Como punto de partida examinaré el modelo planteado por Rosaldo en la década de 1970, el cual se basa en la separación que se produce en sociedades de aldeas sedentarias, en las que se asocia a los hombres con el dominio público y a las mujeres con el privado (1974). Dicha distinción ha sido objeto de muchas críticas, incluyendo a Rosaldo misma (1980), sobre todo en lo referente a que el modelo no se basa en categorías émicas. Luego de tratar y fracasar en la identificación de distinciones de este tipo en sus datos de campo, algunos antropólogos (Bloch y Bloch, 1980; Harris, 1980; Strathern, 1980) concluyeron que dicotomías como natural-cultural y doméstico-público son producto de desarrollos sociales e históricos específicos del Occidente, legados de la filosofía de la Ilustración. Simultáneamente, las críticas marxistas atacaron estas distinciones, sosteniendo que el análisis debería concentrase, más bien, en la producción y la reproducción, que son las "que forman el hogar y otras relaciones sociales" (MacCormack, 2000: 30). Más tarde, Rosaldo aceptaría las críticas:

\footnotetext{
Me parece ahora que el lugar de la mujer en la vida social humana no es en ningún sentido resultado directo de lo que ella haga -0 , todavía menos, una función de lo que ella sea, biológicamente-, sino del significado que sus actividades toman mediante interacciones sociales concretas (1980: 400. Énfasis mío).
}

Parecería que, con esta declaración, la vida útil del modelo de Rosaldo habría llegado a su fin. Sin embargo, a pesar de las severas críticas a que fue sometido, el modelo se utiliza todavía para analizar relaciones de género en diferentes contextos. Tal vigencia teórica se puede explicar por varios factores: uno de ellos es que las explicaciones alternativas que muchos de sus críticos presentaron no demostraron ser más válidas. Además, quizá por cierta concordancia entre las observaciones de las relaciones de género 


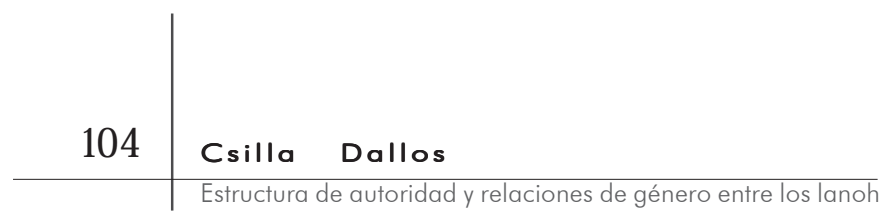

en sociedades sedentarias y los conceptos de Rosaldo, la distinción entre los dominios público y privado continuó utilizándose, incluso por autores que habían presentado explicaciones alternativas $^{1}$. Por último, el modelo ha recobrado vigencia en años recientes. A pesar de admitir las dificultades inherentes a la dicotomía de los dominios público y privado, varios autores, en el volumen compilado por Lugo y Maurer (2000), sostienen también que se lanzó el bebé junto con el agua de la bañera, y que el modelo de Rosaldo, después de todo, parece ser un marco conceptual útil para el análisis de las relaciones de género (2000). Esta resurrección de la teoría de Rosaldo hace muy oportuno discutir un caso de cazadores-recolectores sedentarizados recientemente, en el que los dominios público y privado parecen haberse diferenciado más a partir de la sedentarización, haciendo que las mujeres dejaran de participar en la esfera pública. Sin embargo, y a pesar de los cambios experimentados, las inequidades de género siguen prevaleciendo en la mayoría de los hogares. Al analizar por qué sucede esto, reemplazaré la distinción público-privado que hace Rosaldo por la importancia de aspectos de las dinámicas políticas y de género en grupos recientemente sedentarizados.

\section{LOS LANOH Y SU RELOCALIZACIÓN EN AIR BAH}

N

UESTRA DISCUSIÓN SE BASA EN EL ANÁLISIS DE LA INFORMACIÓN RECOlectada entre los lanoh, uno de los grupos orang asli de la península de Malasia ${ }^{2}$. A pesar de estar administrados por una sola agencia (JOA, y posterior-

1 Por ejemplo, Leacock (1981), desde una explicación marxista de la dominación masculina, sostiene que en pequeñas bandas de cazadores-recolectores no existe diferenciación entre los dominios público y privado.

2. Mi trabajo de campo entre los pobladores de Air Bah, la aldea más importante de reasentamiento de la población lanoh, fue hecho entre diciembre de 1997 y enero de 1999, gracias al financiamiento de un premio de Jóvenes investigadores canadienses, del Centro Internacional de Investigaciones sobre el Desarrollo. Además de investigación participante, los métodos de campo utilizados incluyeron el estudio de la utilización del tiempo, historias de vida y toma de decisiones. mente JHEOA: Jabatan Hal Ehwal Orang Asli, o el departamento de asuntos indígenas) por más de cuarenta años, los grupos orang asli se diferencian entre sí en el mismo grado en que difieren los malayos, los chinos y los indios de los otros habitantes de la Malasia occidental. En términos de sus patrones de subsistencia, los antropólogos han clasificado a los orang asli en poblaciones de 


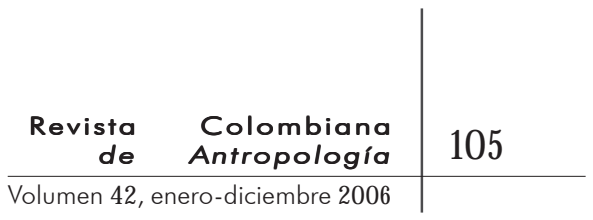

agricultores, horticultores y cazadores-recolectores. Los lanoh son uno de los grupos de cazadores-recolectores forestales que residen en la parte alta del Perak, en el norte de la península, cerca de la frontera con Tailandia (véase el mapa D).

Una premisa de este estudio establece que, a lo largo de su existencia como cazadores-recolectores móviles, los lanoh practicaban una igualdad de género (relativa) ${ }^{3}$. Dicha premisa se basa en el estudio de la vida cotidiana de los lanoh en el periodo anterior a los cambios que suscitó la independencia de Malasia en 1957. Existe fundamento para sostener que se dan, en diferentes sociedades, diferentes grados de igualdad de género. Rosaldo, por ejemplo, basa su argumento en los ilongot, un grupo semisedentario de horticultores en la Filipinas. Sin embargo, los expertos tienden a aceptar que, probablemente, las mayores desigualdades de género se encuentran entre grupos pequeños y móviles de cazadores-recolecto-

3 Las opiniones continúan divididas acerca de si la desigualdad de género es universal o si, por el contrario, existe la posibilidad de igualdad. Leacock (1981) y Ortner (1974) presentan diferentes puntos de vista acerca de este aspecto. Este artículo no busca participar en ese debate, sino analizar cambios en el prestigio, el estatus y la autonomía femeninas. res (Endicott, 1992, 1999, Kent, 1995a). Entre los más igualitarios, de acuerdo con Woodburn (1982) se encuentran los recolectores forestales orang asli de la península Malaya.

La identidad cultural de los lanoh en la época anterior a la independencia ha sido objeto de un prolongado debate entre los antropólogos que se especializan en los grupos orang asli. Un hecho fundamental es que, a diferencia de otros grupos de cazadores-recolectores de la región, como los batek, kintak, kensiu y jahai, que hablan dialectos de la lengua aslian norte, los parientes lingüísticos más cercanos de los lanoh hablan aslian central, el dialecto de uno los mayores grupos de horticultores orang asli: los temiar (Benjamin, 1980). Esta filiación lingüística resalta la condición particular de los lanoh entre los cazadores-recolectores de la región norte de la península malaya. En segundo lugar, se ha propuesto que aun si los lanoh fueron "auténticos" cazadores-recolectores en el pasado, a principios del siglo veinte podía considerárseles agricultores (Benjamin, 1980: 31; K. M. Endicott, 1999). A pesar de estos antecedentes, mis investigaciones demuestran que la forma de vida de los lanoh corresponde a la de otros grupos de cazadores-recolectores de Malasia del norte y Tailandia meridional hasta su sedentarización en la segunda 
MAPA 1

Aldea Air Bah, PeKaK superior,

MALASIA OCCIDENTAL

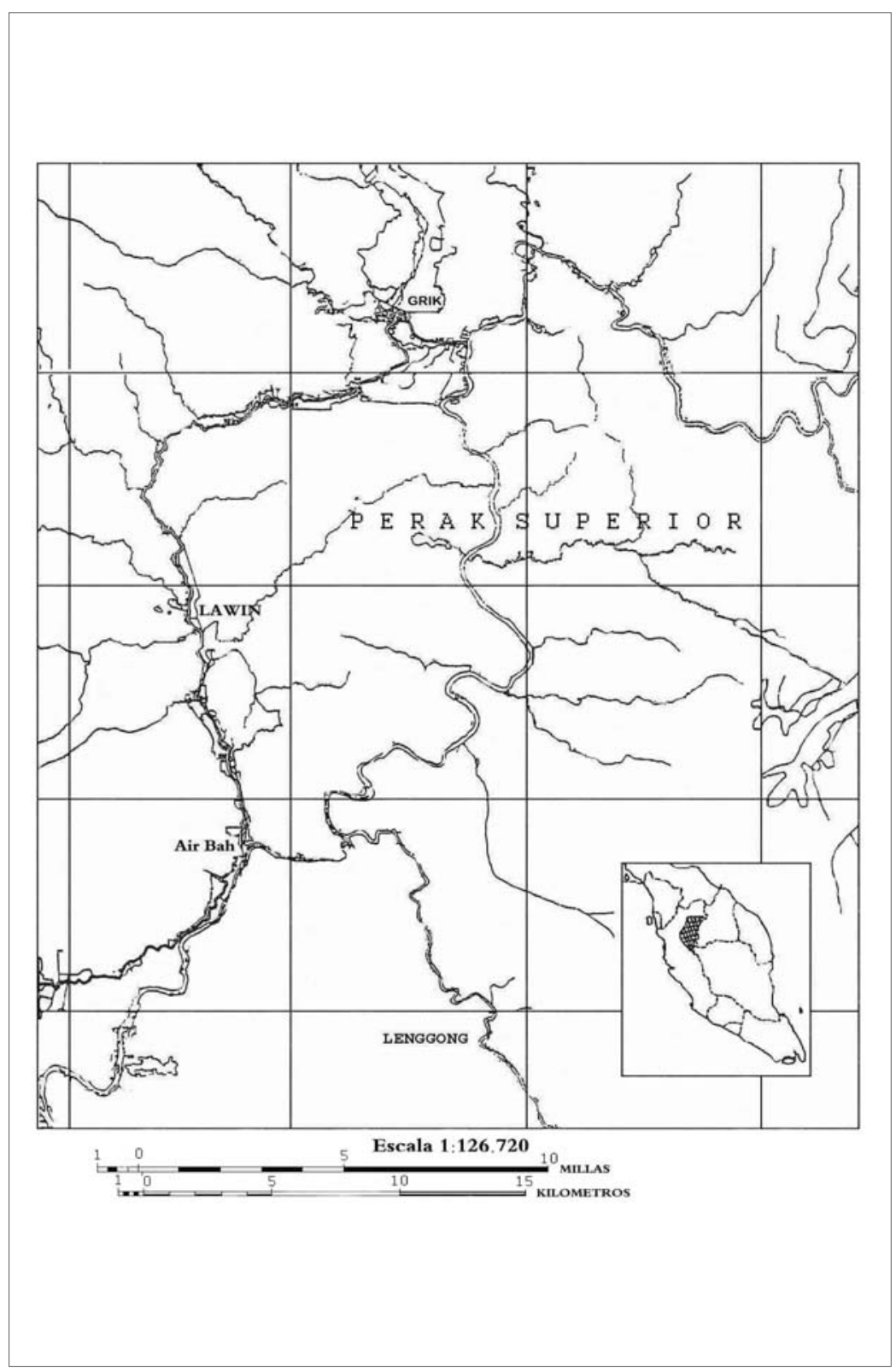




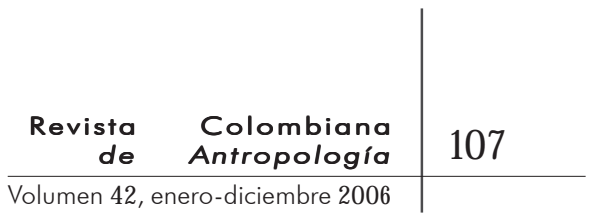

parte de la década de 1970. Los trabajos de Ivor Evans apoyan mi posición. Como el primer etnógrafo de los lanoh, él estudió su vida social, encontrándola similar a la de otros grupos de cazadores-recolectores forestales de la región (1937). Además, aun cuando los lanoh, así como otros grupos de cazadores-recolectores de esta parte de la península, practicaron esporádicamente la agricultura a pequeña escala en el pasado, dichos intentos tropezaron con un serio obstáculo: la necesidad de conservar su movilidad para vender, individualmente, ciertos productos forestales a los agricultores malayos vecinos. Estos requerimientos evitaron, finalmente, que los lanoh se convirtieran en agricultores sedentarios permanentes (Dallos, 2003). Ciertamente, las historias de vida recolectadas en diversos asentamientos lanoh revelan que los informantes vivían como recolectores móviles forestales en su medio tradicional, el valle de Perak, apenas treinta años antes de mi trabajo de campo. Esto, por supuesto, no quiere decir que no sufrieran importantes cambios en su estilo de vida, que incluyeron transformaciones en los énfasis de los diferentes aspectos de sus actividades económicas, inclusive con anterioridad a la segunda parte del siglo veinte. Sin duda, lejos de ser una unidad atemporal, los lonah han sido principalmente afectados por el influjo constante de las influencias culturales y genéticas desde cuando sus antecesores llegaron a la península ${ }^{4}$. A pesar de todo ello, el patrón básico de caza, recolección y recolección en el bosque, que ha involucrado una larga relación con sus vecinos malayos, continuó hasta no hace mucho tiempo.

Aun cuando, por tanto, es claro que los lanoh vivían en pequeños campamentos móviles y aldeas temporales de base, practicando la caza, la recolección y el comercio forestales durante buena parte del siglo veinte, la independencia de Malasia ocasionó

4. Las evidencias arqueológicas sugieren que los orang asli son descendientes directos de los hoabinhians, cazadores y recolectores de amplio espectro, cuyos sitios arqueológicos han sido datados en la península desde el cambios importantes en su modo de vida. Uno de los mayores pro8000 antes de nuestra era (Bellwood, 1985. blemas para los lanoh reside en el deterioro ambiental, causado por la deforestación y por el intenso acaparamiento de la tierra y de otros recursos (Nicholas, 2000). El gobierno malayo trató de solucionar esta situación reagrupando y reasentando a los grupos indígenas que antes eran móviles, imponiéndoles el modo de vida de los agricultores musulmanes. 


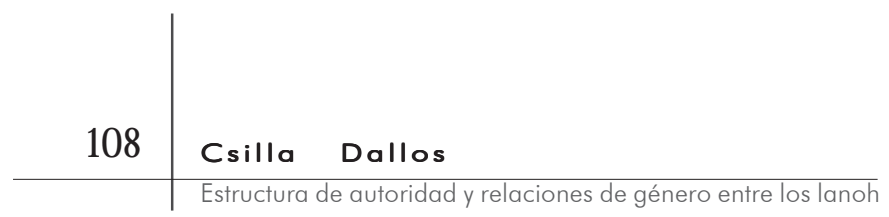

Esta política del gobierno, posterior a la independencia, tiene dos aspectos: por una parte, los orang asli deben ser cambiados porque su movilidad y estilo de vida semi-nómada y su patrón de subsistencia extensivo los ha puesto en la situación de tener que competir por el desarrollo económico. Sus tierras, la selva, es precisamente aquello que requieren y desean los grupos interesados en la explotación maderera, las presas para las hidroeléctricas y las plantaciones (Dentan et al., 1997). Por otra parte, los orang asli deben ser asimilados como parte de la mejoría de la posición de los malayos en el escenario de lo multi-étnico en la economía política de Malasia. En la era poscolonial, los malayos se han encontrado a sí mismos en franca competencia, principalmente con los poderosos grupos chinos. Para optimizar y hacer legítima su posición, aluden a sus derechos como "indígenas" o bumiputera ("princesa del suelo"). Los reclamos de los malayos como indígenas son, por mucho, frágiles; los orang asli, quienes en principio son los verdaderos nativos de la península, son la razón para ello. En relación con los orang asli, los malayos llegaron en grandes cantidades a la península hacia el segundo milenio de la era cristiana, por ello pueden ser considerados también como colonizadores de los orang asli, los nativos que encontraron allí (Dentan et al., 1997: 20-21).

En consecuencia, para el partido político gobernante en Malasia, los orang asli han sido un asunto sensible que continúa poniendo en peligro su imagen como "las gentes originales". Una forma de suavizar esta contradicción es la asimilación de los orang asli a la mayoría malaya y su envolvimiento en el Islam, una característica fundamental que define a los malayos en la península. La integración forzosa al Islam se inició en los últimos años de la década de 1970, con el propósito de integrar a los orang asli "a la sección malaya de la comunidad" (Ministtry of the Interior 1961. 3 En Dentan et al., 1997: 79), y cambió a asimilación, cuyo propósito es la absorción total. Sólo cuando esto último se logre ellos podrán gozar de privilegios semejantes a los de los malayos. Como han resaltado Dentan et al., "muchos malayos ven a los orang asli como "malayos sin terminar", requiriendo únicamente del Islam para estar completos" (Dentan et al., 1997: 81).

Sin duda, en términos del tratamiento de los orang asli existe cierta continuidad entre las políticas coloniales y las poscoloniales. Por ejemplo, los esfuerzos de parte del estado malayo 


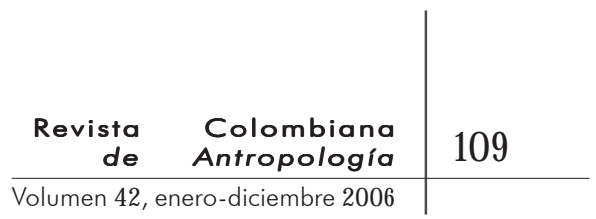

poscolonial por la sedentarización forzosa tienen sus antecedentes en los intentos, de corta duración, realizados por los británicos para reubicar a los orang asli, evitando así que se involucraran en la guerra de insurgencia comunista durante el surgimiento malayo (1948-1960). Además, los orang asli todavía se encuentran gobernados por "el acto de 1954 para los nativos" (Malaysian Government, 1994), que refleja las actitudes paternalistas de la administración colonial británica durante el surgimiento de Malasia. No obstante, los intentos poscoloniales no tienen precedentes en términos del grado en que requieren el cambio económico, cultural y espiritual de los orang asli.

A pesar de que los lanoh han sido relativamente exitosos en la resistencia ante el Islam y la asimilación de los valores malayos, existen fuerzas ante las que han demostrado estar indefensos. Como consecuencia del alto grado de desarrollo y deforestación en el Perak, la movilidad, así como la caza y la recolección, se han visto muy restringidas. Hacia la década de 1980, luego de varios esfuerzos iniciales infructuosos de reasentamiento, los lanoh, quienes habían agotado toda otra alternativa, se establecieron permanentemente en aldeas que les asignó la JHEOA, la agencia gubernamental encargada de los asuntos orang asli. Al disminuir la recolección tradicional de recursos forestales a pequeña escala, que se basaba sobre todo en circuitos individuales, en la actualidad los lanoh participan en actividades especializadas de recolección forestal y ejecutan trabajos por contrato. Otro factor de cambio es que, debido a la sedentarización, la modernización y el abandono de la caza, dependen cada vez más de dinero en efectivo, no sólo para comprar sus alimentos, sino también otros bienes de consumo.

\section{LA SEPARACIÓN DE LOS DOMINIOS PÚBlico Y PRIVAdo EN AIR BAH}

\footnotetext{
PESAR DE QUE, COMO SE HA VISTO, LOS LANOH CONTINÚAN RESIStiendo la influencia islámica en muchos aspectos, así como a Tlas presiones que tienden a convertirlos en agricultores de subsistencia, la vida en Air Bah es muy diferente de lo que era en los campamentos en los cuales fueron pre-reasentados y las aldeas tradicionales lanoh. Primero, la población de las aldeas
} 


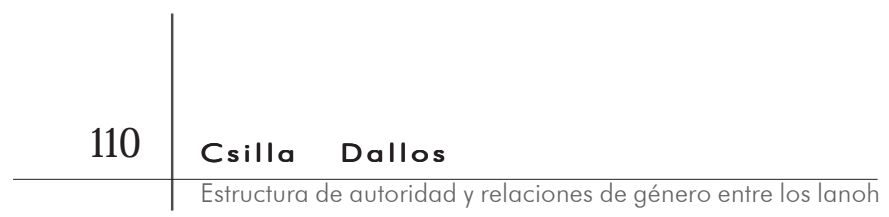

es mayor que la de los antiguos asentamientos lanoh. Según nuestros informantes, la población de las aldeas en las que fueron pre-reasentados raramente excedía los cincuenta habitantes $^{5}$, mientras que la población de Air Bah es de más de doscientos. Esto es resultado parcial de la reagrupación y menor movilidad, de la reducción de la mortalidad infantil, de las consultas médicas más frecuentes, así como de la disminución del uso de prácticas contraceptivas ${ }^{6}$ (Dallos, 2003). Antes de la sedentarización, las mujeres lanoh

5 De acuerdo con Karen Endicott, los camacostumbraban tener, máximo, pamentos móviles batek tienen una población promedio de treinta y cuatro habitantes, divididos entre cinco y ocho familias nucleares (1992: 282).

6. Aparentemente, los lanoh difieren en este aspecto de otros grupos de cazadoresrecolectores de la península malaya. Endicott, por ejemplo, observa que las mujeres barek no usaban ninguna práctica contraceptiva (1992). Las mujeres lanoh, por el contrario, usaban remedios hechos con hierbas para regular su fertilidad. Estas prácticas han sido abandonadas después de la sedentarización. tres niños; actualmente, varias familias en Air Bah cuentan con siete o más. Cerca de la mitad de la población de la aldea tiene menos de veinte años de edad.

El segundo factor, relacionado con el aumento del número de niños por hogar, es la separación de los hombres y las mujeres; los unos y las otras pasan ahora más tiempo en compañía de personas de su mismo sexo. Aun cuando los adolescentes ocasionalmente trabajan, juegan y coquetean en grupos mixtos, los adultos, excepto durante celebraciones ocasionales, raramente pasan tiempo juntos en lugares públicos. Una de las razones para esta separación por género es el cumplimiento de las normas que prescriben la separación en el contexto de la aldea sedentaria. La mayor parte de habitantes de Air Bah están sujetos a estrictas reglas para evitar las relaciones entre los sexos, a tal punto que incluso la sombra de una persona del sexo opuesto debe evitarse. En el pasado, la frecuente movilidad hacía relativamente fácil cumplir con estas reglas. En la actualidad, en el contexto de la aldea sedentaria, la única forma de cumplir con estas reglas es mantenerse en compañía de personas del mismo sexo.

Una segunda razón para la separación de géneros es que ahora las mujeres están sujetas a la aldea, pues sus hogares cuentan con más enseres y niños. Por tanto, la división entre el trabajo doméstico femenino y el trabajo masculino realizado fuera de la aldea es también más pronunciada. Esta división del trabajo antecede hasta cierto punto al sedentarismo, al contrario de lo demostrado por investigaciones sobre otros grupos de cazado- 


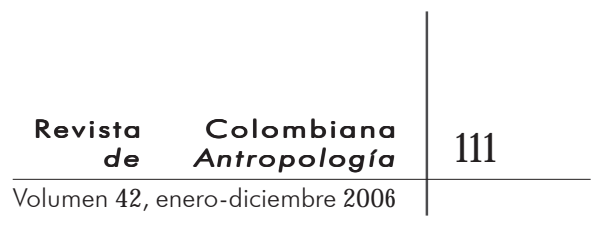

res-recolectores. Kent (1995a), por ejemplo, propone que la desigualdad creciente de género en aldeas de cazadores-recolectores recientemente sedentarizados resulta de los contactos interétnicos más frecuentes que sostienen los hombres. De hecho, en algunos grupos de cazadores-recolectores las mujeres participan en el comercio interétnico. Las mujeres agta no sólo cazan animales de gran tamaño, como cerdos salvajes y venados, sino que también venden la carne seca de estos animales (Estioko-Griffin y Griffin, 1981). Endicott, por su parte, descubrió que las mujeres batek que no tienen niños participan ocasionalmente en la recolección de productos forestales, como el junco, para la venta (1992). Al contrario de dichos grupos, las mujeres lanoh parecen haber estado aisladas de contactos interétnicos hasta poco tiempo antes de la sedentarización. A pesar de que en el pasado acompañaban con frecuencia a los hombres en sus incursiones dentro del bosque, a veces por semanas enteras, ellas recolectaban sólo para su subsistencia, rara vez con propósitos comerciales, y el comercio interétnico era practicado exclusivamente por los hombres. Por esta razón, entre los lanoh los hombres tenían más contacto con personas del exterior, aun antes de su sedentarización.

En la actualidad, las actividades masculinas y femeninas están aún más diferenciadas. Al adoptarse el sedentarismo permanente, las mujeres están menos dispuestas a acompañar a sus parejas por periodos prolongados en sus incursiones en el bosque. Raramente van a Grik, el mercado cercano, ni a otras aldeas. En cambio, ahora pasan la mayor parte del tiempo en la aldea o en sus inmediaciones, penetrando en el bosque sólo durante ocasiones esporádicas, para recolectar productos ${ }^{7}$. En la aldea, además de encargarse de la mayor parte de tareas asociadas con el cultivo de los arrozales $\mathrm{y}$, ocasionalmente, de la tapioca, ellas deben atender a sus hogares y a sus familias, más numerosas ahora. El estudio de la distribución del tiempo revela que las mujeres, exclusivamente, son quienes hacen tareas domésticas en Air $\mathrm{Bah}^{8}$. A pesar de que los hombres a veces cocinan carne, esto no sucede tan a menudo como para ser

7. Aun cuando las mujeres salen ocasionalmente de la aldea para trabajar bajo contrato, sobre todo en una granja china de vegetales cercana, por lo general sus oportunidades de trabajo fuera de la aldea son más reducidas que las de los hombres.

8. Mientras que los hombres pasan $15 \%$ de su tiempo recolectando y $\mathbf{1 6 \%}$ trabajando bajo contrato (31\% en total), las mujeres hacen todo el trabajo doméstico en la aldea $(20 \%$ de su tiempo) y pasan $10 \%$ trabajando bajo contrato fuera de la aldea. 


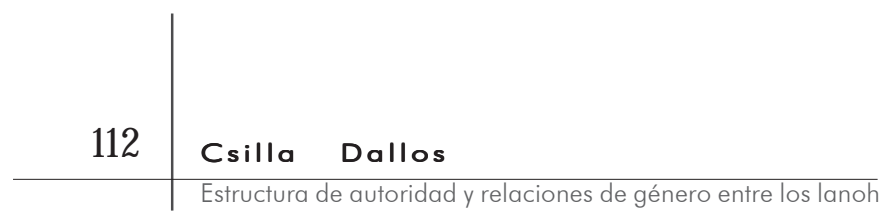

registrado en los datos de asignación del tiempo. Además, ellos no participan ya en el cuidado de los niños al mismo grado que los cazadores-recolectores. En palabras de Karen Endicott, los padres batek "pasan gran parte de su tiempo en el campamento con sus niños. Los padres a menudo bañan a los infantes, atienden a sus necesidades sanitarias y cocinan para ellos" (1992: 283 ). Aun cuando los padres lanoh en Air Bah cargan y sostienen a sus niños a menudo, no atienden a sus necesidades. Las mujeres acompañan a sus niños durante $35 \%$ del tiempo, mientras que los hombres sólo lo hacen durante 3\%.

Con el crecimiento de la aldea, los dominios público y privado se han separado más, separación que dista mucho de ser universal (véanse Harris, 1980; Strathern, 1980). En campamentos reducidos de cazadores-recolectores, prácticamente no existe ninguna distinción entre la esfera pública y la privada. Los lanoh, por ejemplo, vivían en complejos circulares que se prestaban a una participación pública general. Como indica Karen Endicott, en estos asentamientos, "los miembros de la familia duermen, cocinan, comen, guardan sus bienes, descansan y hacen gran parte de su trabajo y del cuidado de los niños dentro y de manera adyacente al albergue" (1992: 283). En estos campamentos los albergues incluían un lugar público en el que el chamán erigía su choza ceremonial ${ }^{9}$, en donde se celebraban todos los eventos públicos. En esta estructura concéntrica, el ámbito

9. De acuerdo con los lanoh, a pesar de que las mujeres tenían conocimiento especializado, poder e influencia como comadronas, los chamanes, como regla, eran hombres. "privado" de los albergues formaba parte del espacio público, ya que, literalmente, era su parte exterior. En este sentido, el espacio público del campamento de los cazadores-recolectores estaba definido por el espacio privado. Además, estos campamentos se caracterizaban por su gran visibilidad, con "albergues abiertos por tres lados, de modo que las actividades de los habitantes quedaban a la vista de todos" (Endicott, 1992: 283).

Con la expansión de los asentamientos, los dominios público y privado se diferenciaron y se separaron más, y los roles domésticos y públicos de los hombres se volvieron más específicos. En la actualidad, en Air Bah el tamaño de los asentamientos y su estructura difieren considerablemente de las aldeas y campamentos lanoh en las cuales fueron pre-reasentados. El dominio público no está más rodeado por el dominio privado. La 


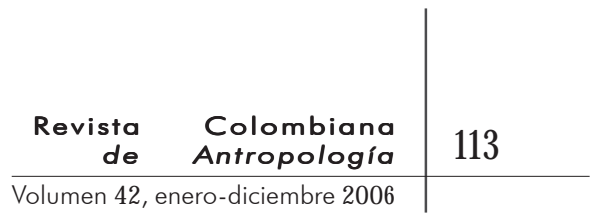

aldea en Air Bah tiene una forma alargada, con las viviendas dispuestas en dos hileras colocadas frente a frente, separadas por cierta distancia. A pesar de que los malayo han erigido una mezquita en un lugar prominente de la aldea, que sirve para recibir a los proselitistas musulmanes y al maestro durante sus visitas esporádicas, así como para alojar al equipo médico durante el periodo mensual de consulta, los hombres lanoh usan este edificio de dos plantas para hacer la siesta y para protegerse del sol del mediodía, en tanto las mujeres lo evitan por completo. Por lo general, ellos se reúnen en un espacio libre en el centro de la aldea, bajo un árbol, pero incluso ese lugar está situado a gran distancia de la mayor parte de las viviendas de la aldea. Por último, las casas de lámina de madera en Air Bah son más grandes y duraderas que los albergues tradicionales. La disposición del asentamiento y el tamaño de las viviendas favorecen la separación de los ámbitos masculinos y femeninos.

La poca distinción entre los dominios público y privado que se da en los campamentos de cazadores-recolectores no parece estar relacionada con la división natural del trabajo entre hombres y mujeres. Yanagisako y Collier sostienen que los conceptos de público y doméstico son "meramente derivaciones y extensiones del mismo hecho natural: la diferencia biológica entre hombres y mujeres" (1987: 15). Por el contrario, en campamentos de cazadores-recolectores la distinción entre los dominios público y privado se basa en la distinción entre el espacio de la pareja y la familia nuclear, por una parte, y el espacio comunal, por otra ${ }^{10}$. En la actualidad, en Air Bah el dominio privado continúa teniendo una connotación de intimidad y de sexualidad. Es ahí donde hombres y mujeres pueden departir libremente. El dominio público, en cierto sentido, es un espacio en el cual las mujeres pasan su tiempo descansando o trabajando en grupos espontáneos. La cocina y el fogón son también áreas semi-públicas, pues por lo general están situadas fuera de las viviendas. A menudo, las viviendas privadas se convierten en espacios públicos, cuando la población entera de la aldea se congrega en alguna de ellas para ver televisión ${ }^{11}$. En otro sentido, sin embargo, el espacio público se convierte en arena política, cuando los hombres organizan reuniones políticas a las que no se invitan mujeres.

10. Maver enfatiza también en el hecho de que el dominio privado es, lo que es más importante, un dominio sexual (2000).

11 Mientras hacía la investigación, cuatro de los quince hogares en Air Bah contaban con aparatos de televisión. A menudo, bienes de consumo como televisores o motocicletas se adquieren gracias a premios de lotería. 


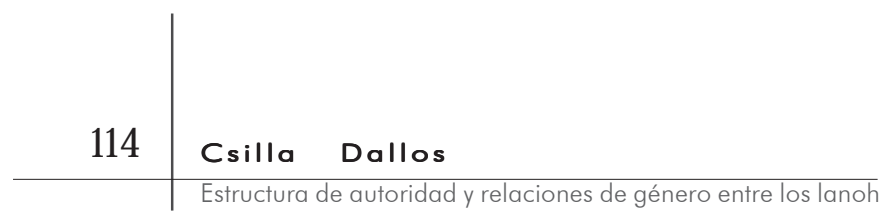

Tanto los hombres como las mujeres tienen diferentes percepciones de estas reuniones públicas. Los hombres no niegan explícitamente a las mujeres el derecho de participar en ellas. En cambio, aparentemente sorprendidos por la idea, se preguntan: "¿Por qué querrían ellas participar en reuniones de hombres?”. Además, a pesar de que ellos reconocen los puntos fuertes de las mujeres, atribuyéndoles cualidades que consideran superiores, por lo general también las consideran menos capaces de abstracción y de expresión oral. Por su parte, las mujeres consideran que estas "serias reuniones políticas masculinas" son ridículas, equiparándolas a juegos infantiles; las ven como una pérdida de tiempo, eventos en los que ninguna mujer en su sano juicio se molestaría en participar. Sin embargo, cuando se sostienen discusiones políticas en las viviendas, las mujeres, sobre todo las de mayor edad, participan libre y abiertamente.

\section{ContinuidAd DE LA IGUALDAD DE GÉNERO EN AIR BAH}

R AJO LA INFLUENCIA DE LA MAYOR DIVISIÓN SEXUAL DEL TRABAJO Y DE la división entre los dominios público y privado, es lícito concluir que las mujeres y los hombres en Air Bah viven más separados que antes; sin embargo, esto no necesariamente reduce su grado de igualdad. Varios factores contribuyen a que las mujeres sigan gozando de una situación igualitaria.

Primero, a pesar de que la agricultura a menudo se asocia con el aumento en la desigualdad de género (Burton y White, 1984), la falta de capital y los requerimientos del trabajo continuo bajo contrato impiden que la mayoría de las familias pueda trabajar en su propia tierra, y aún más, aumentar su producción. En teoría, existe mayor potencial para el cultivo en una aldea sedentaria que en los campamentos móviles y aldeas temporales del pasado. Como parte del acuerdo de reasentamiento firmado con el gobierno malayo, a cada vivienda de Air Bah se le asignó, en la mayor parte de los casos, un mínimo de dos acres de tierra para cultivo. Existe una marcada división sexual del trabajo en la agricultura: las mujeres pasan $10 \%$ de su tiempo cultivando, mientras que los hombres sólo 7\% (Dallos, 2003 185). Los habitantes de la aldea explican que, una vez realizada la siembra, las 


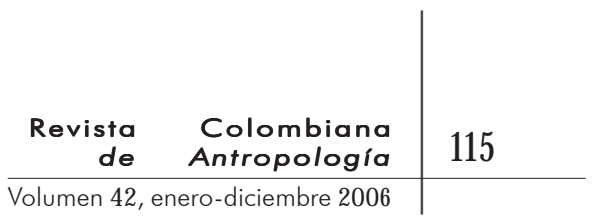

mujeres pueden encargarse de la mayor parte de tareas agrícolas hasta que llega el periodo de la cosecha. Sin embargo, la capacidad de una familia para trabajar la tierra depende de la preparación y de la limpieza del terreno. Este es un trabajo considerado masculino, pero dado que los hombres deben ir a trabajar regularmente fuera de la aldea, rara vez tienen tiempo para hacerlo.

Segundo. El estatus de la mujer es realzado por las reglas de residencia posmatrimoniales matrilocales que prevalecen desde la sedentarización. El patrón de residencia lanoh posmarital era ambilocal-neolocal, con un sesgo inicial matrilocal. Al celebrarse el matrimonio, la joven pareja se mudaba cerca de la familia de la esposa, al menos por algunos meses. Después de cierto tiempo, podían escoger entre quedarse, trasladarse a un lugar más cercano de la familia del esposo o a otro lugar. En la actualidad, debido al sedentarismo permanente, es más probable que la pareja permanezca cerca de la familia de la esposa, lo cual permite que reciba más apoyo de los parientes femeninos. Mujeres de edad avanzada, con mayor poder, se encargan de cuidar a sus nietos o sobrinos, proporcionando compañía constante a sus hijas o hermanas, lo que refuerza aún más la posición de las mujeres jóvenes en sus matrimonios.

El tercer factor es que las mujeres en Air Bah tienen una fuerte convicción de sus derechos en cuanto a sostén económico. A pesar de que permanecen más aisladas del mundo exterior y de que tienen menos oportunidades de ganar dinero, se da por sentado que pueden disponer de sus propias ganancias y de las de sus esposos. Los hombres deben dedicar el dinero que ganan al bienestar de la familia; deben compartir toda ganancia con sus esposas e hijos. Las mujeres, aun cuando a veces gastan dinero en sus niños, no están obligadas a compartir sus ganancias con los hombres adultos que residen en sus hogares. Esta asimetría es aceptada por todos, debido a la obligación masculina de mantener a sus familias. Como resultado, las mujeres, sobre todo las de edad más avanzada, acumulan relativamente grandes sumas de dinero; a menudo, los hombres obtienen préstamos de sus esposas, bajo la estricta obligación de cancelar la deuda más tarde. Las mujeres pueden heredar también propiedades y tierra. Una de las mayores parcelas de Air Bah pertenece a una viuda, quien recibe ayuda de sus parientes masculinos más jóvenes en las tareas agrícolas. a cambio de una parte de la cosecha. 


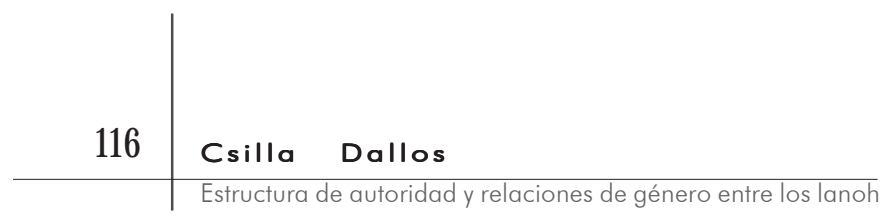

Cuarto. Aun cuando se ha sostenido que la privacidad -además de un aumento de incidentes de violencia contra mujereses un factor que reduce la posición y la autoridad de la mujer en aldeas de cazadores-recolectores recientemente sedentarizados (Draper, 1975a), esto no sucede entre los lanoh de Air Bah. A pesar de los cambios recientes, la violencia es rara y el respeto a la autonomía de los demás que caracteriza a los pueblos orang asli (véase Dentan, 1979, 1992) continúa vigente en la mayor parte de aspectos, debido a la conservación de sus sólidos principios éticos de no-violencia y no-interferencia ${ }^{12}$. El aumento en los incidentes de violencia doméstica entre los cazadores-recolectores recientemente sedentarizados ha sido vinculado a la domesticación de animales (Draper, 1975a) ${ }^{13}$. Los lanoh son conscientes de este vínculo entre el consumo de animales domésti$\cos ^{14}$ y la violencia entre seres humanos (Dallos, 2003) y, en consecuencia, continúan rechazando ambas prácticas.

Por último, las mujeres conservan su igualdad con los hombres porque en Air Bah los líderes masculinos no están investidos de autoridad pública y, por tanto, el dominio público carece de autoridad. En los asentamien-

12. La única excepción a esta continuidad tos circulares del pasado, los líderes, por ejemplo los chamanes, será discutida en este artículo.

13 De acuerdo con Draper (1975a), el aumento en la privacidad de las mujeres y el incremento de la violencia conyugal en albergues cerrados entre los ikung recientemente sedentarizados es causado por la introducción de animales domésticos y la distribución del espacio entre esos animales y las personas. Aunque los lanoh crían gallinas, ellos no interfieren en la autonomía de estos animales y sostienen que los animales que ellos crían no se comen, lo cual resulta en las gallinas vendidas a las aldeas chinas vecinas, $\mathrm{O}$, dado que no tienen restricciones de movimiento, cazadas por gatos, perros o zorros.

14. Aparte de la violencia, la introducción de animales domésticos se ha relacionado también con la reducción en la posición de la mujer (Boserup, 1970; Burton y White, 1984; Taylor, 1996. reclamaban para sí la autoridad al llamar la atención, ya fuera sobre sus personas o sobre sus acciones -magia u oratoria- en el espacio público delimitado por el círculo o semicírculo de los albergues. Con la sedentarización, el contexto de estas demostraciones de autoridad se vio alterado, inicialmente por los cambios acaecidos en la estructura espacial. La sedentarización permanente interfiere con los principios lanoh de liderazgo. Entre los lanoh pre-reasentados, el liderazgo y la autoridad política tradicionales se basaban en el principio de edad relativa. El líder del campamento o aldea era con frecuencia un hombre de edad madura, entre cuarenta y cinco y sesenta años de edad, respetado por los hombres de 


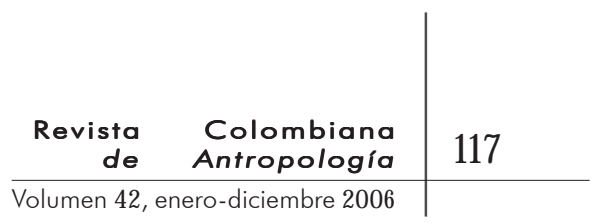

todas las edades ${ }^{15}$. La edad relativa es el principio más importante en las relaciones interpersonales. Aun cuando los habitantes de la aldea no calculan la edad absoluta, ni siquiera la propia, cumplen estrictamente el orden de sucesión y la edad relativa; siempre se puede determinar quien es más viejo o más joven. El cumplimiento con las reglas de la edad relativa es reforzado por el uso de apelativos que diferencian entre parientes viejos y jóvenes, y masculinos y femeninos, incluyendo a los afines ${ }^{16}$. La baja densidad de población, las reglas relacionadas con evitar a ciertas personas y la movilidad frecuente garantiza que ancianos de la misma edad estén presentes en el mismo asentamiento por periodos prolongados. Debido a estos factores, así como al rol de la edad relativa para determinar las relaciones personales, en el pasado los conflictos de liderazgo eran raros.

Sin embargo, en los años que precedieron nuestra investigación la sedentarización permanente $\mathrm{y}$ el aumento de la población debido al reagrupamiento causaron conflictos de liderazgo en Air Bah. En el asentamiento mayor es muy probable que hubiera dos ancianos de la misma edad, cuyas relaciones escapaban, por tanto, al mecanismo regulador de la edad

15 La autoridad masculina disminuye con la edad, una vez pasados los sesenta-sesenta y cinco años, y los ancianos aparentemente pierden tanto influencia como interés en la política. Los ancianos en Air Bah se mostraban retraídos, acostumbraban cazar solos o compartir reminiscencias con hombres de su edad, aun cuando también entretenían a los jóvenes con sus historias. Los jóvenes los consideraban como bromistas o bufones; a menudo, ellos afectaban este rol voluntariamente.

16. Por ejemplo, los parientes más jóvenes y los más viejos son llamados kelong y pe, respectivamente, mientras que el hijo del hermano mayor y menor se llaman kun kelong y kun pe. El hermano mayor de los padres es llamado toy, mientras que la hermana menor del padre se llama mo y el hermano menor bah. Los niños de los hermanos menores y mayores de los padres se llaman kanco y kemuny. Entre parientes afines, el cuñado o cuñada menor es llamado mensaw y el mayor belo. Finalmente, al hermano mayor de la esposa se le llama kenoy. relativa. Dado que ambos se consideraban con derecho a regir la aldea, el conflicto por el liderazgo se convirtió en lucha abierta. El año de nuestra investigación la aldea estaba dividida por este conflicto y ambos líderes se empecinaban por superar al contendiente en todos los campos posibles, especialmente en el de la producción agrícola.

En esta pugna, los líderes rivales pueden contar con todo el apoyo de sus parientes femeninos, en especial de los miembros de su familia nuclear: sus esposas y sus hijas. Las esposas de los líderes forman parte de la contienda, respaldando firmemente las aspiraciones de sus maridos. Ellas realizan trabajo de igual 


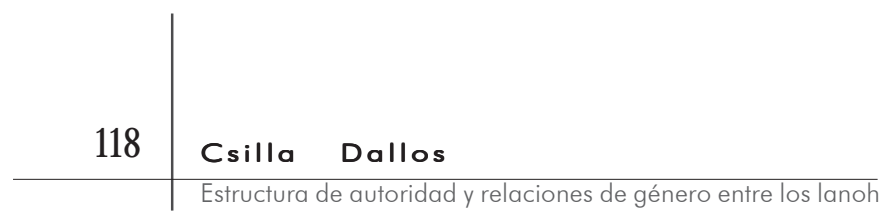

valor al de sus esposos dentro y fuera de la aldea. Por ejemplo, una de las esposas de los líderes organiza y dirige el grupo de trabajadoras por contrato, la otra acompaña periódicamente a su marido, ayudándolo en las tareas de un contrato excepcionalmente bien pagado. Los líderes reciben también ayuda de sus hijas, aun cuando tal apoyo es menos pronunciado. Los hogares de los líderes rivales cuentan con la ayuda de las hijas mayores para las tareas domésticas, permitiendo que sus madres trabajen fuera del hogar y de la aldea. Estos comportamientos corroboran las observaciones de Draper (1975), quien sostiene que las mujeres, en especial las más jóvenes, son educadas de manera más estricta -con mayores obligaciones en el hogar- y que esta tendencia se ha acentuado en las aldeas recientemente sedentarizadas. En los hogares de los líderes rivales, los padres aprovechan dicha tendencia para aumentar la productividad del hogar. En una aldea en la que la escogencia de pareja matrimonial raramente es influenciada por la familia, ambas familias han creado mitos paralelos -cuyos miembros no se comunican entre sí- para justificar por qué, debido a sus propias actitudes y acciones, estas dos muchachas se han convertido en parejas indeseables y no pueden casarse con los hombres que las cortejan ${ }^{17}$. Retener a sus hijas en el hogar añade valiosa fuerza de trabajo a los hogares de ambos líderes rivales.

El apoyo de los parientes masculinos a los líderes en pugna es mucho menos marcado. Esto se debe a que la naturaleza individual de la recolección tradicional y el respeto por la autono-

17. De acuerdo con esta historia, un día, el hijo menor del líder se enfermó y se llamó a un curandero. Cuando el muchacho se repuso, el hombre Semai no quiso aceptar ningún pago de sus padres. En cambio, quiso más bien casarse con las dos hijas mayores del líder. Al rechazarlo, las muchachas cometieron selantap, un pecado. Debido a esto, nadie más puede casarse con ellas.

18. Hombres más viejos que los líderes en pugna han dejado la recolección comercial y el trabajo bajo contrato, pero no participan tampoco en el cultivo. Sólo hacen la recolección ligera de productos forestales menores, como bambú para hacer cerbatanas y juguetes, así como plantas medicinales, y cazan de vez en cuando. mía individual de los demás continúan contrarrestando la autoridad basada en la edad en Air Bah. Además, actualmente los líderes y sus parientes masculinos participan en diferentes actividades económicas. Los hombres más jóvenes se ocupan sobre todo de la recolección y trabajan por contrato fuera de la aldea, mientras que los más viejos han abandonado estas duras actividades y se dedican más a cultivar la tierra ${ }^{18}$. Como tales, los líderes ejercen 


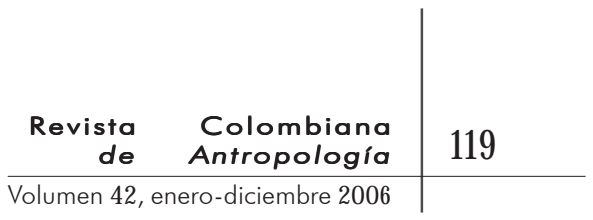

poco control sobre las actividades de sus parientes masculinos más jóvenes. Aún más, al contrario de las mujeres, quienes departen sobre todo con sus parientes, los hombres más jóvenes o más viejos que los líderes establecen alianzas que cruzan los límites de parentesco, y no están dispuestos a sacrificar estos vínculos para beneficiar a ninguno de los líderes. Como resultado, estos no cuentan con ayuda significativa de sus parientes masculinos. Por ejemplo, el cultivo de la tierra de los líderes requiere un intenso trabajo de deshierbe, que sólo puede hacerse una vez que el terreno ha sido desbrozado con maquinaria pesada por la compañía que le había comprado sus árboles de caucho. De esta forma, aunque los parientes masculinos brindan apoyo moral a los ancianos de sus respectivos grupos de parentesco, este apoyo no es suficiente para generar facciones políticas ni grupos organizados basados en el parentesco ${ }^{19}$.

En consecuencia, los líderes contendientes en Air Bah participan en un tipo de política que podría llamarse más privada que pública. Al faltarles apoyo decidido se retiran a sus respectivos hogares y, de mal humor, rehúsan participar en la toma pública de

19. Esto tiene influencia, a su vez, sobre la capacidad de la mayoría de las mujeres para conservar su posición. De acuerdo con Sacks (1979, el surgimiento de grupos de parentesco organizados sustrae a las mujeres del control de su propia producción, facilitando la dominación masculina.

decisiones. Esto afecta la capacidad de los lanoh para encargarse de las necesidades de organización, que ahora son mayores que en las pequeñas aldeas y campamentos del pasado. Ocasionalmente, hombres de menor edad que los líderes contendientes tratan de organizar reuniones para encargarse de estos asuntos. Sin embargo, debido a que sus roles están prescritos por la edad, no son aún elegibles para ejercer cargos públicos. Para que actividades comunales, como la compra de gasolina, la reparación del generador y de las cañerías, los funerales o las fiestas de la recolección puedan realizarse efectivamente deben ser organizadas y financiadas por forasteros. Como resultado, a pesar de la mayor desigualdad, muchos en la aldea desean que se imponga autoridad. Los habitantes de la aldea añoran los viejos tiempos, en los que los líderes lanoh tenían más poder e imponían más respeto. Es probable que estas percepciones se deban más a la falta de autoridad pública que a los atributos personales de los líderes. La falta de autoridad pública afecta también la condición de la mujer en 


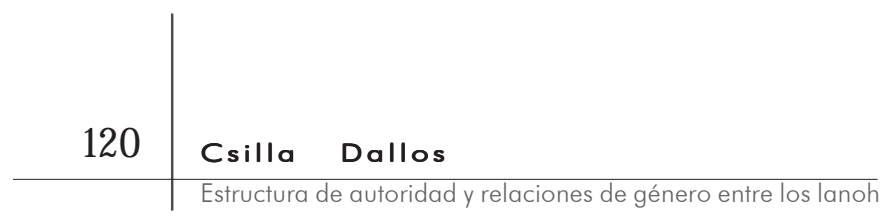

Air Bah. El dominio público, aun cuando está más diferenciado de la esfera doméstica que en el pasado, no tiene implicaciones políticas, de modo que algunas mujeres en Air Bah han llegado a tener más influencia política que la mayor parte de hombres.

Se reconoce que son particularmente las mujeres de mayor edad las que controlan solapadamente la política de la aldea; ellas ejercen influencia sobre sus parientes femeninas -hijas, sobrinas, hermanas y nietas-, quienes, a su vez, presionan a sus maridos. Estas mujeres mayores influyen también sobre la lucha por el liderazgo. Quizá por la falta de apoyo masculino, los líderes dependen de sus alianzas con estas mujeres. Un ejemplo elocuente es la alianza entre uno de los líderes y una mujer, hermana mayor de la esposa del líder. A pesar de las reglas que les impiden comunicarse directamente o mencionar sus nombres respectivos, ella coordina sus objetivos y medios con los del líder contendiente mediante los miembros femeninos del hogar del líder. Es un secreto a voces que son ellas quienes deciden acerca del futuro político y la organización de la aldea; con su poder contribuyen también a que surjan desigualdades entre las familias, al excluir algunas menos populares de la comunicación y el acceso a los recursos, la información y los eventos comunitarios por medio de rumores, lo que refuerza la posición de su propio grupo de parentesco.

Por esta razón, los significados culturales que en diferentes circunstancias podrían servir para denigrar a las mujeres, no afectan su condición en Air Bah. Es por esto también que los hombres consideran la presunta falta de capacidad de abstracción e interés en "asuntos serios de política" como una característica positiva, y que no tiene ninguna implicación para el estatus femenino. Esto explica por qué las actuaciones de los hombres en reuniones públicas, realizadas con gran pompa, son consideradas como farsas por las mujeres, quienes tienen medios efectivos para influenciar la toma de decisiones por medio de la esfera privada. Resumiendo, es por esto que la diferenciación de los roles de género y la diferenciación de los dominios público y privado no implican desigualdad de género en Air Bah. 


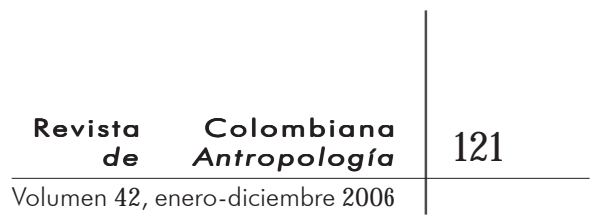

\section{Discusión y CONCLUSIONES}

STE ARTÍCULO TIENE IMPLICACIONES PARA LA TEORÍA DEL DESARROLLO - histórico de la desigualdad en las relaciones de género. ReL conozco que algunos aspectos del cambio social experimentado por los lanoh son específicos de su sociedad, y que no es posible separarlos de las contingencias históricas y de los efectos de la modernización acaecida a fines del siglo veinte. Estas circunstancias específicas incluyen la sedentarización (forzada), la participación en el trabajo bajo contrato y la dependencia creciente del ingreso de los hombres. Además, cabe observar que, con todo y la importancia de la limitada autoridad de los líderes de Air Bah, antes de volverse sedentarios los lanoh habían sido cazadores-recolectores y comerciantes. Hasta la fecha no se sabe con certeza cómo afecta el comercio con pueblos vecinos de pastores y agricultores las relaciones sociales de los cazadores-recolectores a pequeña escala (Dallos, 2003). Por tanto, es limitado el grado al cual podemos generalizar las conclusiones de este caso a instancias prehistóricas e históricas de sedentarismo.

Igualmente, algunos aspectos de los cambios que se dieron en Air Bah, como el aumento de la población, la composición, el patrón y el tamaño del asentamiento, la competencia por el liderazgo, así como la dependencia creciente de la agricultura, son comunes a diversos contextos históricos y geográficos. Aldeas recién sedentarizadas, tanto históricas como prehistóricas, pueden haberse visto sometidas a problemas y desafíos similares. En consecuencia, las conclusiones de este caso pueden servir de punto de partida para el estudio del surgimiento de las desigualdades.

El análisis del cambio social en Air Bah muestra que la separación de los dominios público y privado, a pesar de ser, en algunos casos, sintomática de relaciones de género desiguales, no es ni causa ni "marco estructural subyacente" (Lamphere, 1993 75) de la desigualdad de género. En Air Bah, a pesar de la separación de los dominios público y privado, la igualdad de género continúa prevaleciendo, excepto en el caso de las dos mujeres que están subordinadas a una unidad corporativa y cuya autonomía ha sido claramente restringida. La cuestión es, ¿cómo interpretar estos resultados a la luz de las teorías de la desigualdad de género? 


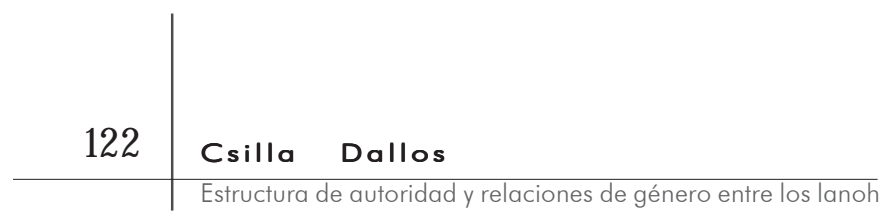

Durante largo tiempo, las explicaciones históricas de la desigualdad de género estuvieron dominadas por autores marxistas (Engels, [1891] 1972; Leacock, 1978, 1981; MacCormack, 2000). No cabe duda de que dichos estudios han hecho importantes avances, señalando los factores que intervienen en el desarrollo histórico de la desigualdad de género; sin embargo, sus interpretaciones no dejan de presentar problemas y sus conclusiones necesitan ser reevaluadas (Lewellen, 2003). Estas explicaciones sostienen que el surgimiento de la producción como valor de cambio y el control de la propiedad privada conduce a la desigualdad de género. La existencia de cazadores-recolectores que participan en el comercio y están expuestos al desarrollo en aldeas recientemente sedentarizadas, como Air Bah, muestra que la universalidad del postulado anterior está en tela de juicio.

El caso analizado en el presente artículo indica también que no es plausible que los significados e interpretaciones culturales de hechos biológicos sean necesariamente la causa de la desigualdad de género. Una explicación más convincente es que la desigualdad es el resultado de una complejidad social creciente que, a su vez, se precipita por cambios demográficos y en los patrones de asentamiento. Nuestro estudio sugiere además que las relaciones de género entre cazadores-recolectores recientemente sedentarizados no siempre cambian como resultado de la aculturación o de la adopción de las costumbres de los agricultores vecinos (e. g. Kent, 1995). Al contrario, el caso de Air Bah sugiere que los desarrollos internos radican en cambios en la estructura de vida. Además, usando el lenguaje de la teoría de la complejidad, estos cambios están enraizados en la organización y en los patrones de interacción tradicionales de los cazadores-recolectores.

El análisis de estos cambios nos permite comprender la dinámica de lo que pudiera considerarse como un primer paso hacia la complejidad social. Cambios en la dinámica de población y de patrones de asentamiento llevan a competir por el liderazgo; esta, a su vez, se convierte en conflicto que resulta en la auto exacerbación de las cualidades personales y en el aumento de la producción doméstica. Un segundo factor por considerar es que las dinámicas que se dan dentro de los hogares en competición indican que el conflicto de liderazgo puede tener un papel, no sólo en el desarrollo de los grupos organizados de parentesco, sino en el aumento de la desigualdad de género en comunidades recientemente sedentarizadas. 


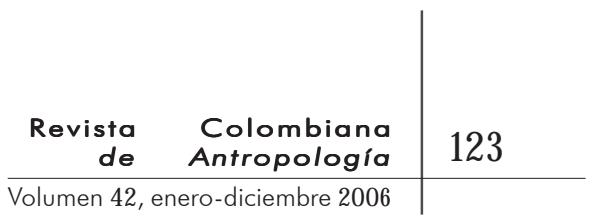

Tercero. El estudio, una vez más, ha señalado la importancia de los roles sociales y los estatus para comprender los cambios en las relaciones de género en comunidades recientemente sedentarizadas (Sacks, 1979). Al buscar las raíces de la desigualdad de género es necesario diferenciar entre los roles que las mujeres adoptan como hermanas, madres, hijas y esposas; y considerar también, como sugiere Draper, el proceso de socialización (1975). Este enfoque podría reforzar el hecho de que mientras las mujeres mayores pueden conservar o aumentar su estatus y las esposas continúan gozando de igualdad, es entre las tímidas y sometidas hijas que radica la desigualdad de género en aldeas nuevamente sedentarizadas. También habrá que reconocer el papel de las mujeres mayores en el desarrollo de la desigualdad de género. No es claro aún de qué manera el tratamiento de las hermanas influencia los valores, percepciones y expectativas de los hombres jóvenes que crecen en esos hogares. ¿No estarían ellos siendo condicionados para esperar la sumisión y el sacrificio de sus esposas, como resultado del ejemplo que ven en sus hogares?

La comprensión de las dinámicas sociales de Air Bah también proporciona al analista escenarios alternativos con diferentes consecuencias para las relaciones de género. Cuando los ancianos en competencia pueden contar con suficiente apoyo masculino y donde, como resultado, grupos organizados de parentesco están surgiendo, la competencia por el liderazgo puede desplazarse a la esfera pública. En este caso es fácil ver cómo la prepotencia y la megalomanía de los líderes en pugna le darían especial importancia a la esfera pública, en detrimento de la privada, y cómo, por asociación, los hombres comenzarían a considerarse más importantes que las mujeres, quienes permanecerían relegadas a un segundo plano, dedicándose calladamente a sus actividades. Además, este caso no sólo contribuye a comprender cómo las relaciones de género entre cazadoresrecolectores recientemente sedentarizados pueden volverse desiguales; indica también que este no es necesariamente el caso. En este sentido, consideramos que al no darse la competencia por el liderazgo ni su resultante complejidad social, comunidades sedentarias relativamente grandes y populosas pueden continuar teniendo relaciones igualitarias de género.

Una implicación adicional de este estudio es que si negamos que la separación de las esferas pública y privada sea la causa de la 


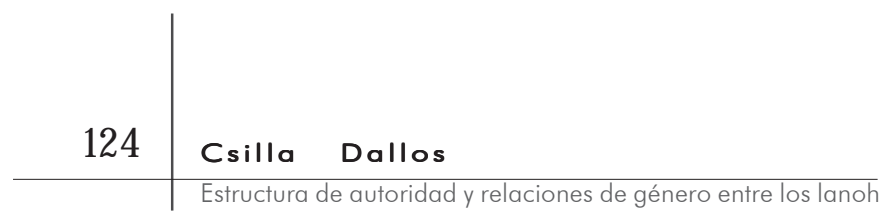

desigualdad de género, la solución propuesta por Rosaldo, a saber, la inclusión de los hombres en las tareas domésticas, queda en duda. Aun si incluir a los hombres en las tareas domésticas permite reducir la carga de trabajo de las mujeres y fomentar una vida doméstica más democrática, es posible que también acarree nuevos problemas. Debe tenerse en cuenta que aun cuando en el seno de los grupos de cazadores-recolectores los hombres se involucran en el cuidado de los niños, no lo hacen a expensas de su participación en los asuntos públicos. De hecho, si aceptamos que hombres y mujeres poseen diferentes estrategias y prioridades reproductivas, como muchos autores han sostenido (Graves-Brown, 1996; Hawkes, 1996; Ecuyer-Dab y Robert, 2004), podemos entonces considerar la posibilidad de que hombres y mujeres tengan también diferentes motivaciones y objetivos al encargarse del cuidado de los niños. En grupos reducidos, el cuidado de los niños podría ser, para los hombres, una forma de demostrar sus capacidades políticas, de exhibir su sentido de responsabilidad y su capacidad de cuidar de los demás ${ }^{20}$. De esta forma, en tales circunstancias, el cuidado de los niños, que para

20. El término "exhibirse" es tomado de la tesis de Kristen Hawkes (1991) acerca del significado probable de la caza masculina en grupos reducidos de cazadores-recolectores. Sin embargo, no hay razón para no extender este razonamiento a toda clase de trabajo ejecutado por hombres. relaciones de género en sociedades a pequeña escala. Los resultados presentados en este artículo sugieren que para comprender la desigualdad de género en esas sociedades es posible que debamos empezar a analizar las relaciones entre hombres, y no sólo entre hombres y mujeres. Este podría ser un asunto para futuras investigaciones.

\section{BibliografíA}

Bellwood, Peter. 1985 Prehistory of the Indo-Malaysian Archipelago. Academic Press. Sydney.

Benjamin, G. 1980. "Semang, Senoi, Malay: Culture-History, Kinship and Consciousness in the Malay Peninsula”. Manuscrito inédito. 


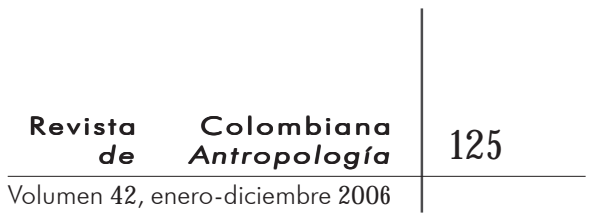

Blanton, R. E. 1995 "The Cultural Foundations of Inequality in Households". En T. D. Price y G. M. Feinman (eds.). Foundations of Social Inequality. Plenum. Nueva York.

Bцосн, M. y J. H. Bцосн. 1980. "Women and the Dialectics of Nature in Eighteen-century French Thought". En C. MacCormack y M. Strathern (eds.). Nature, Culture, and Gender. Cambridge University Press. Cambridge.

Boserup, E. 1970. Women's Role in Economic Development. St. Martin's Press. Nueva York.

Burton, M. L. y D. R. White. 1984. "Sexual Division of Labor in Agriculture". American Anthropologist. 86.

Collier, J. y S. J. Yanagisako (eds.). 1987. Gender and Kinship: Essays Towards a Unified Analysis. Stanford University Press. Stanford.

Dallos, C. 2003 "Identity and Opportunity: Asymmetrical Household Integration among the Lanoh, Newly Sedentary Hunter-Gatherers and Forest Collectors of Peninsular Malaysia”. Tesis doctoral inédita. McGill University. Montreal.

Dentan, R. K. 1992. "The Rise, Maintenance, and Destruction of Peaceable Polity: A Preliminary Essay in Political Ecology". En J. Silverberg y J. P. Gray (eds.). Aggression and Peacefulness in Humans and Other Primates. Oxford University Press. Oxford.

1979. The Semai. A Nonviolent People of Malaya. Holt, Rinehart and Winston. Nueva York.

Dentan, R. K., K. Endicott, A. Gomes y M. B. Hooker. 1997. Malaysia and the "Original People": A Case Study of the Impact of Development on Indigenous Peoples. Allyn and Bacon. Boston.

Di Leonardo, M. (ed.). 1991 Gender at the Crossroads of Knowledge: Feminist Anthropology in the Post-Modern Era. University of California Press. Berkeley.

Draper, P. 1992. "Room to Maneuver: !Kung Women Cope with Men". En D. Counts et al. (eds.). Sanctions and Sanctuary: Cultural Perspectives on the Beating of Wives. Westview Press. Boulder.

1975a. "!Kung Women: Contrasts in Sexual Egalitarianism in Foraging and Sedentary Contexts". En R. Reiter (ed.). Toward an Anthropology of Women. Monthly Review Press. Nueva York.

\section{Ethnologist. 2.}

Ecuyer-Dab, I. y M. Robert. 2004. "Have Sex Differences in Spatial Ability Evolved from Male Competition for Mating and Female Concern for Survival?”. Cognition. 91 


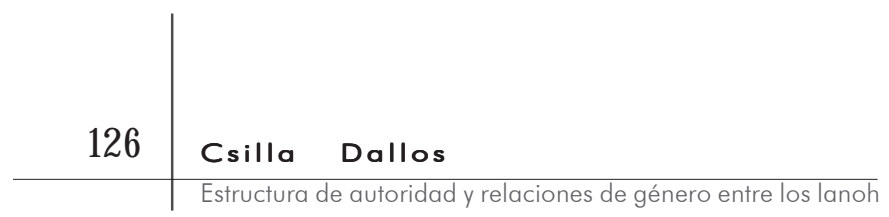

Endicott, K. L. 1999. “Gender Relations in Hunter-Gatherer Societies”. En R. B. Lee y R. Daly (eds.). The Cambridge Encyclopedia of Hunters and Gatherers. Cambridge University Press. Cambridge.

. 1992. "Fathering in an Egalitarian Society". En B. S. Hewlett (ed.). Father-Child Relations: Cultural and Biosocial Contexts. Aldine de Gruyter. Nueva York.

EndicotT, K. M. 1999. “Introduction: Southeast Asia”. En R. B. Lee y R. Daly (eds.). The Cambridge Encyclopedia of Hunters and Gatherers. Cambridge University Press. Cambridge.

Engels, F. (1891) 1972. The Origin of the Family, Private Property and the State. International Publishers. Nueva York.

Estioko-Griffin, A. y P. B. Griffin. 1981 "Woman the Hunter: The Agta". En F. Dahlberg (ed.). Woman the Gatherer. Yale University Press. New Haven.

Evans, I. H. N. 1937. The Negritos of Malaya. Cambridge University Press. Cambridge (reimpreso en $\mathbf{1 9 6 8}$ por Frank Cass and Co. Ltd. Londres).

Graves-Brown, P. 1996. “Their Commonwealths Are not as We Supposed: Sex, Gender and Material Culture in Human Evolution”. En J. Steele y S. Shennan (eds.). The Archaeology of Human Ancestry: Power, Sex and Tradition. Routledge. Londres.

GrinkeR, R. R. 1992. "History and Hierarchy in Hunter-Gatherer Studies”. American Ethnologist. 19.

Harris, O. 1980. "The Power of Signs: Gender, Culture and the Wild in the Bolivian Andes”. En C. P. MacCormack y M. Strathern (eds.). Nature, Culture and Gender. Cambridge University Press. Cambridge.

Hawkes, K. 1996. "Foraging Differences between Men and Women: Behavioural Ecology and the Sexual Division of Labour". En J. Steele y S. Shennan (eds.). The Archaeology of Human Ancestry: Power, Sex and Tradition. Routledge. Londres.

1991 "Showing off. Test of an Hypothesis about Men's Goraging Goals”. Ethology and Sociobiology. 12.

Kelly, R. 1995 The Foraging Spectrum: Diversity in Hunter-Gatherer Lifeways. Smithsonian Institution. Washington.

KEnT, S. 1995a. "Does Sedentarization Promote Gender Inequality? A Case Study from the Kalahari”. Journal of the Royal Anthropological Institute. 1

19950. "Unstable Households in a Stable Community: The Organization of a Recently Sedentary Kalahari Community”. American Anthropologist. 97. 


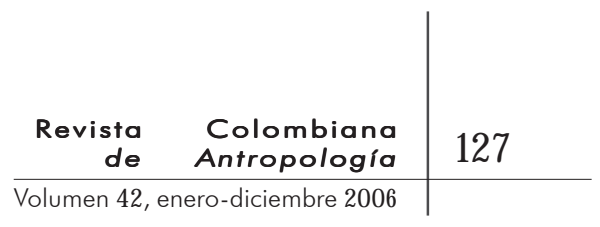

LAMPHERE, L. 1993 "The Domestic Sphere of Women and the Public World of Men: The Strengths and Limitations of an Anthropological Dichotomy”. En C. B. Brettell y C. F. Sargent (eds.). Gender in Cross-Cultural Perspective. Prentice Hall. Englewood Cliffs.

LeAcock, E. 1981 Myths of Male Dominance. Monthly Review Press. Nueva York.

- 1978. "Women's Status in Egalitarian Society: Implications for Social Evolution”. Current Anthropology. 19.

Lewellen, T. C. 2003 Political Anthropology. Praeger. Westport.

Lugo, A. y B. Maurer. 2000. “The Legacy of Michelle Rosaldo: Politics and Gender in Modern Societies”. En A. Lugo y B. Maurer (eds.). Gender Matters: Rereading Michelle Z. Rosaldo. The University of Michigan Press. Ann Arbor.

MacCormack, C. 2000. "Land, Labor, and Gender". En A. Lugo y B. Maurer (eds.). Gender Matters: Rereading Michelle Z. Rosaldo. The University of Michigan Press. Ann Arbor.

Malaysian Government. 1994. Akta Orang Asli 1954/Aboriginal Peoples Act 1954 (with all amendments up to June 1994). Malaysian Government. Kuala Lumpur.

Maurer, B. 2000. "Sexualities and Separate Spheres: Gender, Sexual Identity, and Work in Dominica and Beyond”. En A. Lugo y B. Maurer (eds.). Gender Matters: Rereading Michelle Z. Rosaldo. The University of Michigan Press. Ann Arbor.

Ministry of the InTERIOR. 1961 Statement of Policy Regarding the Administration of the Aborigine Peoples of the Federation of Malaya. Ministry of the Interior. Kuala Lumpur.

Nicholas, C. 2000. The Orang Asli and the Contest for Resources: Indigenous Politics, Development and Identity in Peninsular Malaysia. IWGIA. Copenague. Documento 95

Ortner, S. B. 1974. “Is Female to Male as Nature is to Culture?”. En M. Z. Rosaldo y L. Lamphere (eds.). Woman, Culture and Society. Stanford University Press. Stanford.

Rosaldo, M. Z. 1980. “The Use and Abuse of Anthropology: Reflections on Feminism and Cross-Cultural Understanding”. Signs. 5

1974. "Woman, Culture and Society: A Theoretical Overview”. En M. Z. Rosaldo y L. Lamphere (eds.). Woman, Culture and Society. Stanford University Press. Stanford.

SACKS, K. 1979. Sisters and Wives: The Past and Future of Sexual Equality. Greenwood Press. Westport. 


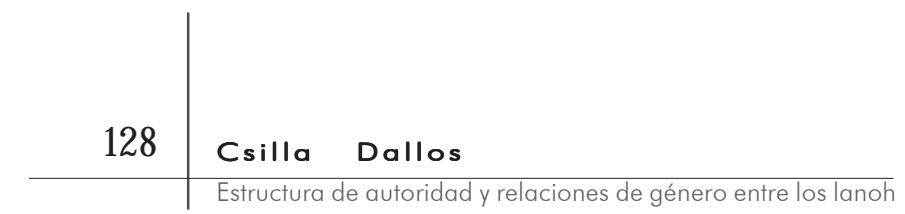

Sнотт, M. J. 1992. "On Recent Trends in the Anthropology of Foragers: Kalahari Revisionism and its Archaeological Implications". Man. 27.

Strathern, M. 1980. "No Nature, No Culture: The Hagen Case". En C. P. MacCormack y M. Strathern (eds.). Nature, Culture and Gender. Cambridge University Press. Cambridge.

TAYlor, T. 1996. The Prehistory of Sex. Bantam Books. Nueva York.

Woodburn, J. 1982. "Egalitarian Societies". Man. 17 (3).

YANAgisako, S. J. y J. F. Collier. 1987. "Toward a Unified Analysis of Gender and Kinship”. En J. F. Collier y S. J. Yanagisako (eds.). Gender and Kinship: Essays Towards a Unified Analysis. Stanford University Press. Stanford.

Recibido: 17 de abril de 2006.

Aprobado: 24 de junio de 2006. 